\title{
Microfluidic Technology in Vascular Research
}

\author{
A. D. van der Meer, ${ }^{1}$ A. A. Poot, ${ }^{1}$ M. H. G. Duits, ${ }^{2}$ J. Feijen, ${ }^{1}$ and I. Vermes ${ }^{1}$ \\ ${ }^{1}$ Polymer Chemistry and Biomaterials Group, Faculty of Science and Technology, University of Twente, \\ P.O. Box 217, 7500 AE Enschede, The Netherlands \\ ${ }^{2}$ Physics of Complex Fluids Group, Faculty of Science and Technology, University of Twente, \\ P.O. Box 217, 7500 AE Enschede, The Netherlands
}

Correspondence should be addressed to A. D. van der Meer, a.d.vandermeer@utwente.nl

Received 26 May 2009; Revised 3 August 2009; Accepted 24 August 2009

Recommended by Manuela Martins-Green

Vascular cell biology is an area of research with great biomedical relevance. Vascular dysfunction is involved in major diseases such as atherosclerosis, diabetes, and cancer. However, when studying vascular cell biology in the laboratory, it is difficult to mimic the dynamic, three-dimensional microenvironment that is found in vivo. Microfluidic technology offers unique possibilities to overcome this difficulty. In this review, an overview of the recent applications of microfluidic technology in the field of vascular biological research will be given. Examples of how microfluidics can be used to generate shear stresses, growth factor gradients, cocultures, and migration assays will be provided. The use of microfluidic devices in studying three-dimensional models of vascular tissue will be discussed. It is concluded that microfluidic technology offers great possibilities to systematically study vascular cell biology with setups that more closely mimic the in vivo situation than those that are generated with conventional methods.

Copyright (C 2009 A. D. van der Meer et al. This is an open access article distributed under the Creative Commons Attribution License, which permits unrestricted use, distribution, and reproduction in any medium, provided the original work is properly cited.

\section{Introduction}

Vascular science is an active area of research. Scientists worldwide are trying to unravel the mechanisms that determine vascular function and dysfunction. Important objects of study in this field of research include the maintenance of vascular tone [1], regulation of inflammation [2], sprouting of new blood vessels [3], regulation of cell survival [4], and the differentiation of stem cells into vascular tissue [5]. Vascular science is a field with a strong translational focus, combining results from fundamental molecular and cell biology with in vitro models of blood vessels and in vivo tests to develop insight in vascular physiology and treatment of disease. Vascular dysfunction is an important factor in major diseases like atherosclerosis [6], cancer [7], and diabetes [8]. The basis for understanding the functioning of blood vessels lies in understanding its building blocks, the vascular cells. Therefore, a lot of research is focused on how endothelial cells or smooth muscle cells react to relevant biological, chemical, or physical cues in vitro. Usually, this work is carried out by using conventional methods, culturing cells of animal or human origin in wells-plates, subjecting them to the aforementioned stimuli, and analyzing the outcome by biological or biochemical techniques. However, in vivo dynamic conditions are present: vascular endothelial cells are constantly subjected to shear stress caused by the flowing blood [9], while smooth muscle cells are stretched because of distension of the blood vessel during the cardiac cycle [10]. Moreover, vascular cells are embedded in a three-dimensional environment consisting of an elastic extracellular matrix [11], other cells [12], and flowing blood, with its platelets [13], red blood cells [14], and leukocytes [15] (Figure 1). Both the three-dimensional environment and the dynamic mechanical changes with each cardiac cycle are very important factors in vascular cell functioning. It is advantageous to design laboratory setups that allow researchers to include these factors and control the relevant parameters. The main challenge when building such setups is that they should still be easy to assemble, handle, and combine with conventional analysis techniques.

In the recent years, the field of microfluidic technology has gained much scientific interest among biologists, biochemists, and biophysicists (Figure 2). We feel that microfluidic technology holds great promise to overcome 


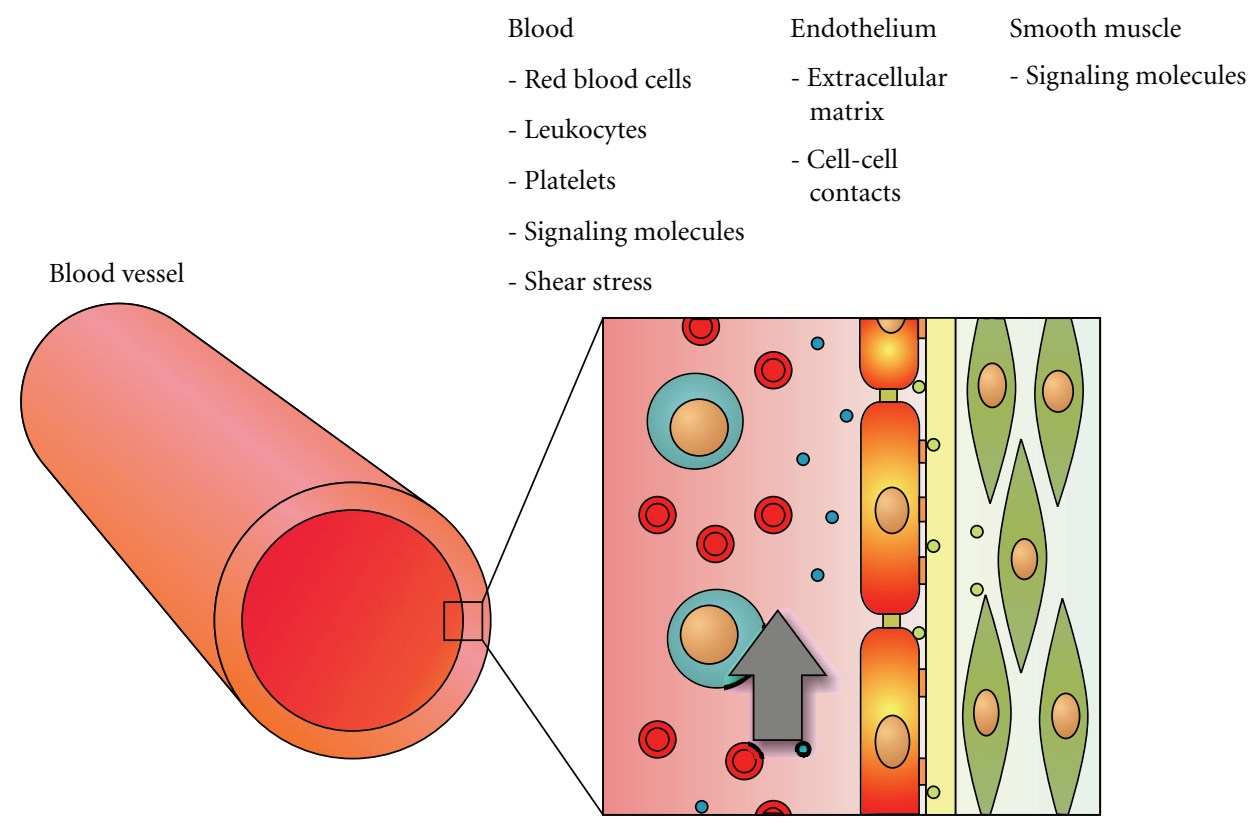

FIGURE 1: Schematic overview of a blood vessel and the endothelial cell microenvironment. The inner wall of a blood vessel (left) consists of a layer of endothelial cells that are embedded in a three-dimensional microenvironment (right). This environment consists of cell biological, biochemical, and physical stimuli, such as red and white blood cells, signaling molecules, and shear stress, respectively. Mimicking this complex microenvironment in vitro is a major challenge in vascular research.

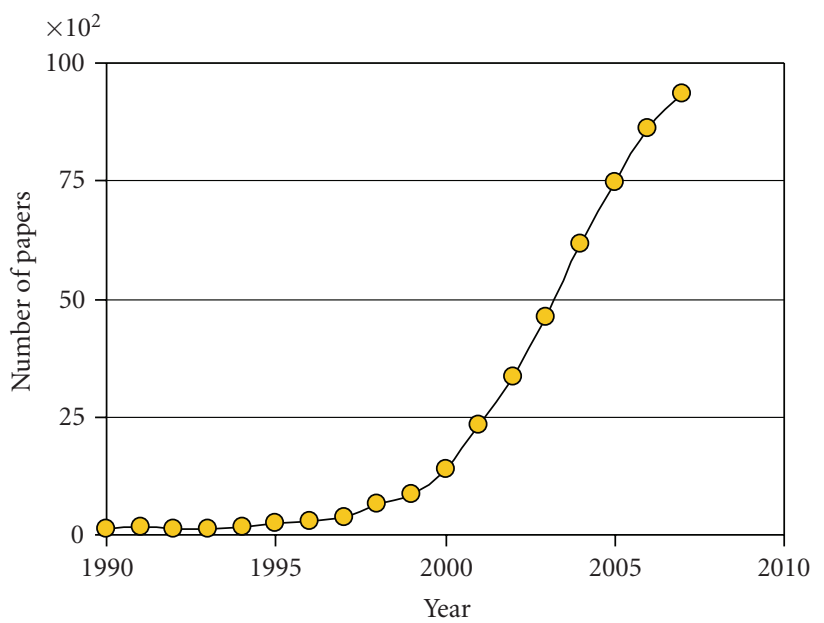

FIGURE 2: Graph of the amount of papers containing the keyword "microfluidic" that were published per year as determined with Google Scholar. A more than 30-fold increase in the yearly number of scientific publications with this particular keyword can be observed in the decade between 1995 and 2005.

the challenge of performing in vitro experiments with more physiologically realistic setups that are still simple enough to be used in everyday laboratory practice. Moreover, microfluidic technology allows for increasing scale and parallelization of current research, leading to more comprehensive insights into cell and tissue physiology. The advantages of microfluidic technology for cell culture in general have been reviewed elsewhere $[16,17]$. In this review, we will focus specifically on the application of microfluidic devices in vascular cell biology research.

\section{Microfluidic Technology}

2.1. Fabrication. Microfluidic technology deals with the design, fabrication, and application of devices for manipulation of fluids on the micrometer scale. Typically, the sizes of features in these devices range from several micrometers to a few hundred micrometers. The amounts of fluid that are manipulated inside these devices are typically in the picoliter to nanoliter range. Microfluidic devices can be fabricated using metal, glass, or polymer materials. Most devices that are used in combination with cell biological research are made of glass or the silicone rubber polydimethylsiloxane (PDMS), because these materials are cheap, biocompatible, and transparent.

Because all microfluidic studies that are discussed in this review use microfluidic devices of PDMS (sometimes combined with glass components), the process of producing these devices will be described shortly (see also Figure 3). PDMS devices are produced by soft lithography replica molding [18], which means that the devices are elastic replicas of a stiff, reusable mold. The process starts by producing the stiff mold with the desired structures. The mold is usually made of silicon with micrometer-size structures produced either by plasma-etching of the silicon plate or by building on top of the plate with the epoxy-based, photo-crosslinkable polymer SU-8. A mixture of PDMS oligomers is poured on top of this mold, allowed to solidify by crosslinking, and then peeled off from the mold. In order to create sealed channels, the surface of the PDMS replica is activated with oxygen plasma and bound to a PDMS or glass surface. Holes can be punctured to reach the closed channel structure and tubing can be connected to manipulate fluid inside the channels. The silicon master-molds need to be produced in 


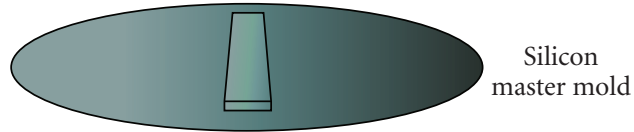

(a)

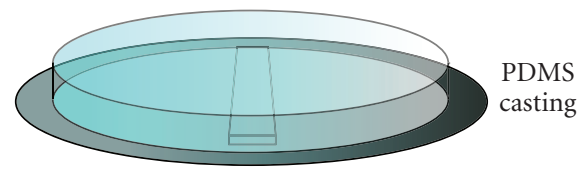

(b)

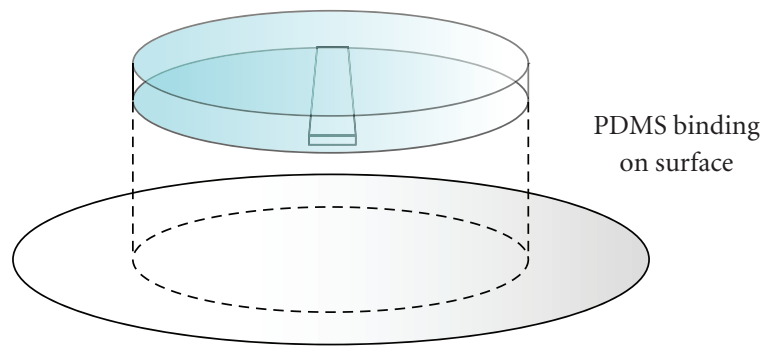

(c)

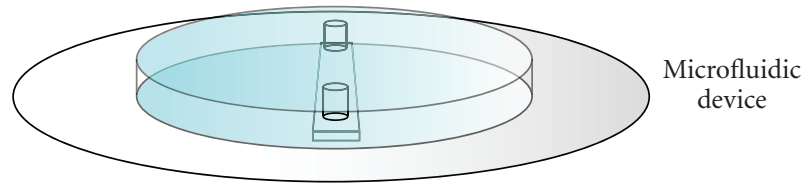

(d)

FIGURE 3: Schematic overview of the fabrication of PDMS chips. (a) The process starts by fabricating a silicon master moldwith typical dimensions of 10 centimeter in diameter and 0.5 millimeter thickness - with microfluidic structures on top. (b) A viscous mixture of PDMS oligomers and crosslinker is poured on top of the mold and allowed to form a flexible, crosslinked network. (c) The slab of PDMS is peeled off of the mold and bound to a glass or PDMS surface to produce closed microfluidic channels. (d) The microfluidic device is ready to be used. Prior to binding to the surface, holes can be punched in the slab of PDMS to reach the resulting microfluidic channel.

a clean room, but replica molding can be performed under standard laboratory conditions. Once the master-mold has been created, producing new microfluidic devices by this method takes only a few hours. Because the materials are cheap, microfluidic devices can be discarded after every experiment.

2.2. Cells and Microfluidic Technology. Generally speaking, PDMS microfluidic devices offer a number of distinct advantages over conventional techniques for cell culturing, manipulation, and analysis. The main feature of microfluidic devices that makes them suitable for use in cell biology is that they are smaller than conventional setups (for an impression of the size of a microfluidic channel, see Figure 4(a)). Because of this small size, only limited amounts of cells, media, and reagents are needed. This leads to a number of significant benefits. First of all, if experiments are to be conducted with rare primary cell material or expensive drugs, it is quite advantageous to use only small quantities of these valuable materials. Secondly, if cultures are to be maintained under conditions of constant fluid flow, small sizes are a considerable advantage. In conventional bioreactors, cell culture medium is usually collected and re-used after it has passed through the cell culture chamber. The medium is then completely replaced every few days. In microfluidic devices, a constant flux of fresh medium can be used, because the volumes involved are orders of magnitude smaller. The third benefit is that the small, planar and transparent microfluidic setups are easily combined with bright field and fluorescence microscopy or spectroscopy, because they fit easily on stages of conventional microscopes. This facilitates monitoring cell behavior for long periods and with high magnification during the experiment.

It is important to realize that cells that are cultured inside microfluidic devices need to be subjected to a constant flux of fresh medium. When the small volumes in the cellcontaining devices would be left under static conditions, nutrients would be depleted quickly, whereas waste products would increase to undesirable concentrations. The fact that constant refreshment of medium is needed may seem cumbersome at first glance. However, under physiological conditions, all cell types need a flux of nutrients and waste products. The flow conditions in microfluidic devices mimic this process more closely than in vitro culturing in wells plates [19].

Because of the small dimensions of microfluidic channels, fluid flow is fully laminar, meaning that the flow patterns are completely predictable and turbulent mixing does not occur. In some applications, such as microreactors, which require mixing of different reagents, this laminar flow pattern is an obstacle that has to be overcome. However, the laminar nature of the fluid flow can also be used to perform unique experiments that are difficult or nearly impossible to perform with conventional methods. Most of these experiments rely on parallel fluid flows: if two streams of fluid enter the chip in a parallel fashion, the two streams will remain separated and mixing of them will only occur by diffusion. Thus, the degree of mixing can be tuned by changing the flow rate: the higher the flow rate, the shorter the residence time inside the device, the less the streams mix. Therefore, as long as flow rates are sufficiently high, cells on one side of the device can be treated with one substance, whereas cells on the other side are treated with another substance. As a matter of fact, even two sides of a single cell can be treated in this way [20].

Another important main feature of microfluidic technology is that it is suitable for high-throughput, comprehensive studies of cell biology. This means that the effect of multiple factors and parameters on cell functioning can be screened in one assay. Increasing throughput is an active area of research in the field of microfluidics. Efforts are made to merge microfluidic technology with microarray and microtitre plate technology [21-23]. Also, researchers are dedicated to integrate multiple steps, such as cell culturing, lysis, and analysis in one device. Numerous examples of this parallel and serial microfluidic biochemical analysis, also known as 


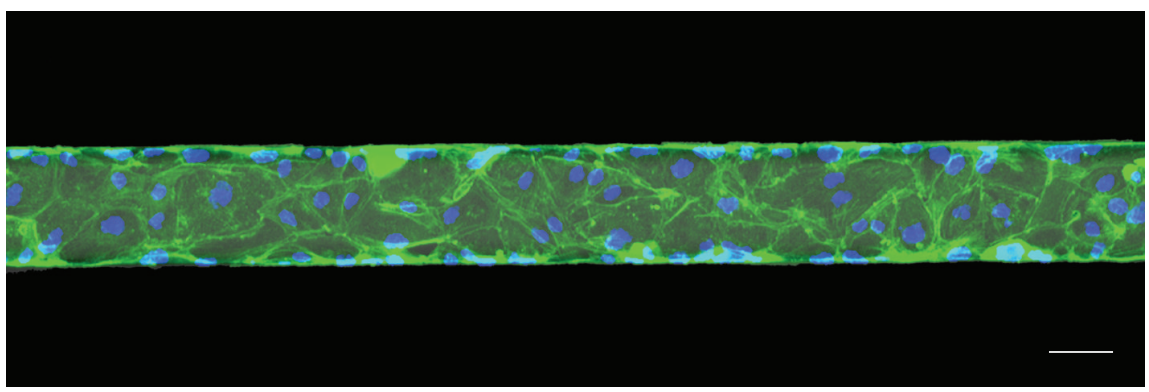

(a)
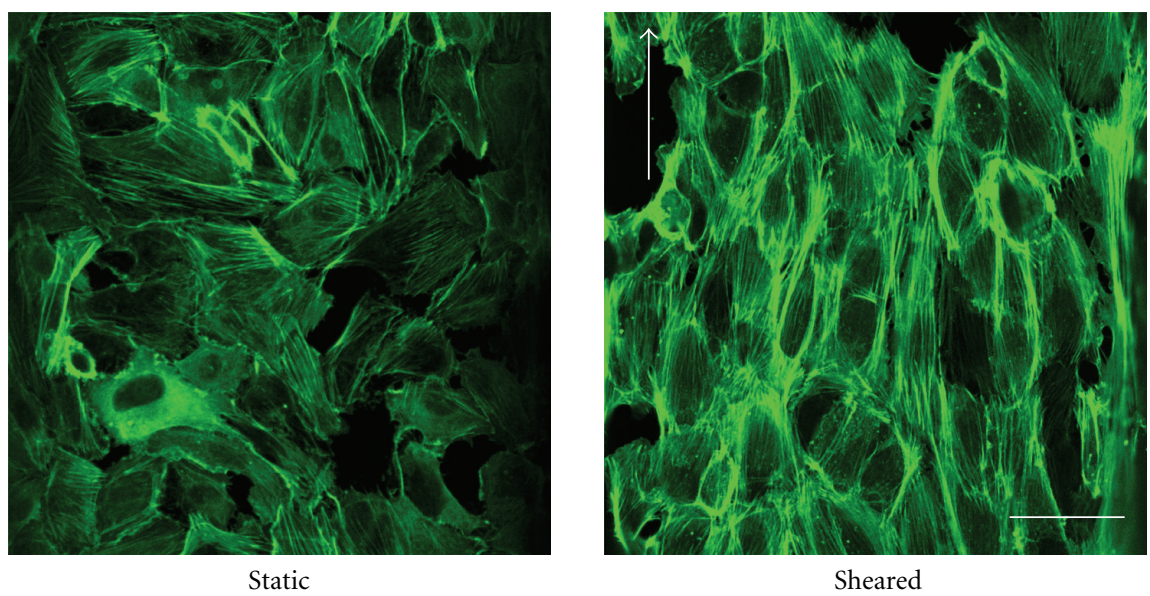

(b)

FIGURE 4: Endothelial cells in a microfluidic channel. (a) Human umbilical vein endothelial cells were cultured in a microfluidic channel. When reaching confluence, the cells were fixated with paraformaldehyde and stained for actin filaments (green) and nuclei (blue). Scale bar is $50 \mu \mathrm{m}$. (b) Endothelial cells that are subjected to physiological levels of shear stress inside microfluidic channels reorient their actin cytoskeleton to align with the direction of fluid flow (right). Scale bars are $50 \mu \mathrm{m}$.

lab-on-a-chip, have already been reported and are starting to be implemented in cell-containing microfluidic devices [16].

\section{Microfluidic Technology and Vascular Cells}

3.1. The Endothelial Mechanoresponse. Vascular endothelial cells are highly responsive to shear stress that is caused by the flow of fluid over their surface. This shear stress is the result of the presence of a fluid velocity gradient in the cross section of a tube. The velocity of the fluid next to the walls is zero, whereas the velocity is maximal in the center of the channel. The steeper this gradient, the higher the shear forces that act on the vessel wall. The biological response to this mechanical stimulus - the endothelial mechanoresponsehas been found to be a key process in preventing vascular disease [24]. The mechanoresponse is usually studied in vitro by subjecting endothelial cells to shear stress in parallel plate flow chambers. Microfluidic devices can be considered as miniaturized versions of these setups. Because shear stress is proportional to flow rate and inversely proportional to channel dimensions, only low flow rates are needed in microfluidic channels to mimic the high shear stresses found in the human body. Song et al. [25] took advantage of this fact by designing a microfluidic device that can subject endothelial cells to physiological levels of shear stress in multiple parallel channels. They showed that a flow rate of less than $200 \mu \mathrm{L}$ per hour is already enough to make the sheared endothelial cells elongate and orient in the direction of the flow, which is a prominent feature of the endothelial mechanoresponse that is also found in vivo. This reorientation is also reflected in the actin cytoskeleton of the cells. In our laboratory, we subjected cells to a shear stress of $1 \mathrm{~Pa}$ for 12 hours and then stained the actin filaments with phalloidin-FITC. Most filaments were aligned and oriented in the flow direction (Figure 4(b)). Recently, Tkachenko et al. [26] also reported the design of a microfluidic device that allows for real-time tracking of endothelial cells that are subjected to shear stress. They could generate shear stresses ranging from 0.01 to 0.9 $\mathrm{Pa}$ in parallel channels, using flow rates in the range of several milliliters per hour. In contrast, flow rates are in the order of hundreds of milliliters per hour for the conventional, larger, parallel plate flow chambers. Because of the small volumes of reagents that are needed, and the potential parallelized design of microfluidic devices, they are an ideal platform for screening of compounds that may have an impact on the mechanoresponse. We have recently developed such an assay, in which the morphological rearrangements of endothelial cells are used to quantify the mechanoresponse. Using this assay, the impact of inhibitory drugs on the mechanoresponse can be detected (paper submitted). 


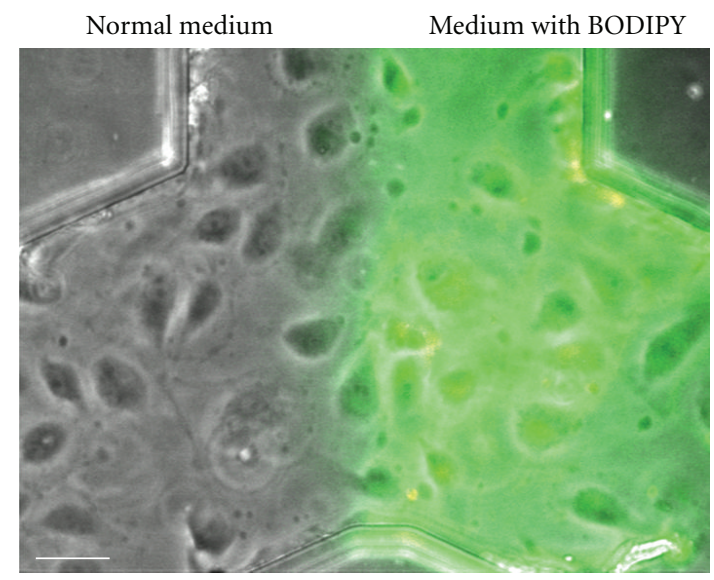

(a)

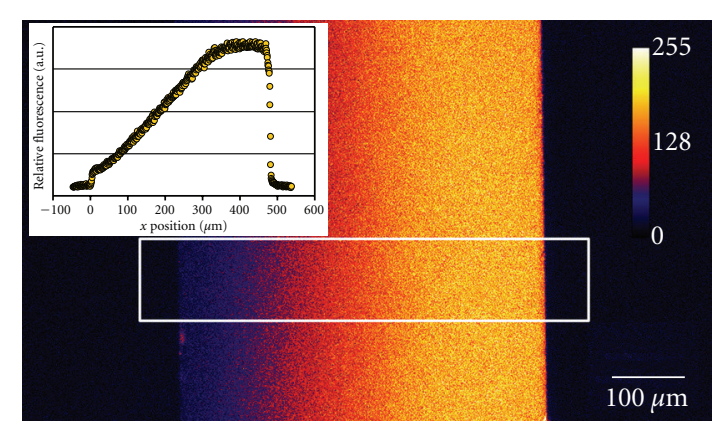

(b)

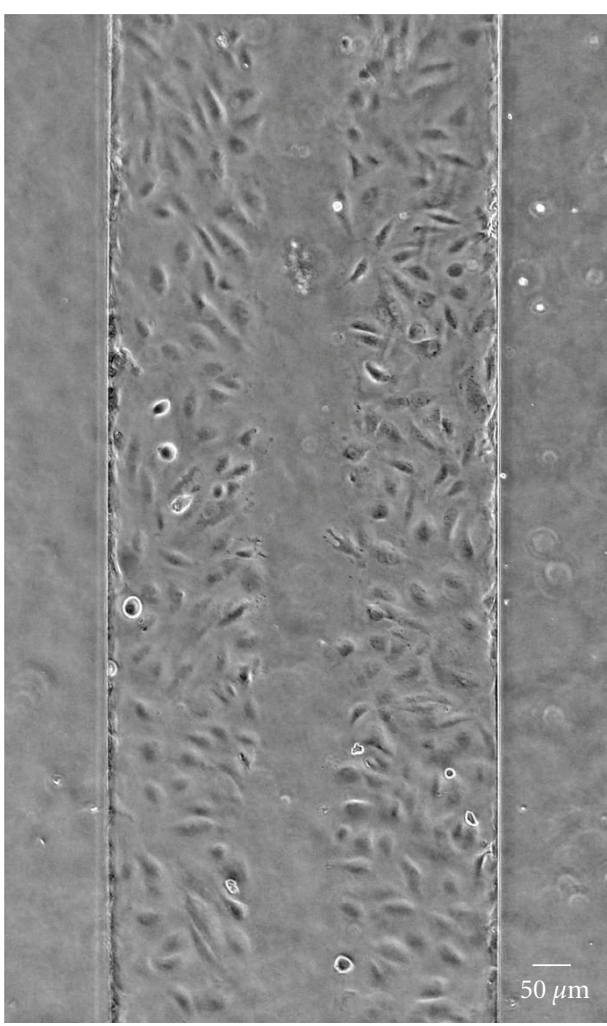

(c)

FIGURE 5: Uses of parallel fluid flows inside microfluidic channels. (a) Parts of the microfluidic channel can be treated differently by pumping two types of media into the two inlets. In this case, a fluorescent label was added to one of the parallel fluid streams. Flow is from bottom to top; scale bar is $50 \mu \mathrm{m}$. (b) When flow rates are sufficiently low, media reside in the channel long enough for diffusion to take place. This phenomenon can be used to generate and maintain steady gradients in a channel. In this case, three parallel inlet streams were used, containing $0 \mu \mathrm{g} / \mathrm{mL}, 5 \mu \mathrm{g} / \mathrm{mL}$, and $10 \mu \mathrm{g} / \mathrm{mL}$ dextran-rhodamine, respectively. When quantifying the fluorescence over the width of the channel, an almost linear gradient can be observed (white square box in the image, plotted in the inset). (c) By using parallel flows, the middle part of the channel was treated with trypsin. As a result, endothelial cells in the middle of the channel are selectively removed, creating an artificial wound. The closing of this wound can be followed over time to quantify cell migration rates.

Another well-known effect of applying shear stress to endothelial cells is the release of the vasodilatant, nitric oxide [27]. Microfluidic assays have already been reported that can detect the production of nitric oxide in response to chemical stimuli amperometrically [28] or by fluorescence [29]. This provides researchers with an interesting tool to study nitric oxide release in response to both mechanical and chemical stimuli.

When increasing the flow rate and the resultant shear stress, microfluidic devices can also be used to study the adhesion strength of endothelial cells to their underlying substrate. Young et al. [30] performed such an experiment with endothelial cells of different origins and two types of matrix proteins. When the cells were subjected to a shear stress that is about ten times higher than typical physiological values, a certain percentage of cells detached from the surface. In this manner, it was possible to give a semiquantitative indication of the strength of adhesion of different cells on different substrates. These types of experiments used to be performed with large, parallel plate shear devices that consumed large amounts of media, cells, and reagents [31]. Downscaling of these setups to micrometer dimensions is a clear advantage.

3.2. Migration Assays. As discussed earlier, multiple parallel fluid flows can be introduced in one microfluidic channel. Transport of components from one flow to the other only occurs by diffusion (Figure 5(a)). If flow rates are low, there is sufficient time for the parallel streams to exchange components. If one of the streams contains a drug or active compound, stable gradients can be generated by taking advantage of this diffusion. An example of such a gradient that was produced in our laboratory is shown in Figure 5(b). There are a number of studies that show how this phenomenon can be used when experimenting with vascular cells. Most of these studies focus on migration of vascular cells in response to gradients of physical or biochemical cues. Studying and understanding cell migration is important, because it is a process involved in embryogenesis, wound healing, and tumorigenesis. 
Barkefors et al. [32] studied migration of endothelial cells in gradients of vascular endothelial growth factor (VEGF). They designed a device with three inlets, generating three parallel fluid streams in the main channel. When VEGF was added to the middle stream, an increasing gradient from the sides of the channel towards the middle was generated. The steepness of this gradient could be tuned by adjusting the flow rates: the slower the flow rate, the longer the residence time in the channel, the more time there is for VEGF to diffuse, and the more shallow the gradient becomes. When endothelial cells were cultured in this stable gradient of VEGF, they preferentially migrated towards the middle of the channel. Because the researchers had control over the shape of the gradient, they could show that steep gradients induce faster migration. Moreover, it was found that endothelial cells migrated fastest in gradients from 0 to $50 \mathrm{ng} / \mathrm{mL}$, whereas they were not able to sense gradients from 50 to $100 \mathrm{ng} / \mathrm{mL}$ due to saturation of the available receptors.

Biochemical cues, such as the growth factors used in this study, are not the only relevant stimuli for vascular cell migration. In an elegant study, Zaari et al. [33] showed that smooth muscle cells tend to migrate towards mechanically stiffer underlying substrates. To reach this conclusion, the authors designed a microfluidic device that could generate a gradient of crosslinker, mixed with a solution of acrylamide. A layer of gel with a gradient of stiffness was produced by crosslinking the mixture with UV light into a polyacrylamide network. When these gels were taken out of the microfluidic devices and smooth muscle cells were seeded on them, all cells tended to migrate towards the side of the gel with higher stiffness.

Apart from migration assays that rely on gradients, the parallel laminar fluid streams can also be used to bring the most conventional migration assay to a microfluidic scale. This assay is the scratch assay or wound healing assay. It works by growing cells in a monolayer, artificially creating a scratch and then following how this scratch is closed by directed migration of the surrounding cells. In a microfluidic device, the artificial scratch can be generated by adding the serine protease trypsin to one of the parallel fluid streams. When one side of the channel has been cleared of cells by trypsinization, the migration of the remaining cells can be followed over time to quantify directed migration. So far, this assay has only been published with data on fibroblasts [34], but work in our group has shown that it is also possible with endothelial cells (Figure 5(c)). The advantage of carrying out this assay in a microfluidic device is that it can be more easily combined with stimuli such as shear stress or growth factor gradients.

3.3. Cell Interactions. The principle of parallel streams is not just useful in studies of cell migration. It can also be used to pattern cells inside a microfluidic device. This is important when interactions between cells are the object of study. Micrometer-scale patterning of cells can be achieved by stamping adhesive proteins on a substrate [35] or by temporarily confining cells in a microfluidic device until they adhere, after which the device is removed from the surface [36]. By using parallel streams, cells can be patterned without the need of removing the microfluidic device afterwards. When adding one cell type to one stream and another type to the parallel stream, cells can be cocultured in direct contact with each other inside a microfluidic device [37]. For vascular research, this method could be used to pattern endothelial cells and smooth muscle cells in one device. The planar nature of microfluidic devices would provide great opportunities for studying interactions between these cell types. In literature, there are numerous reports of microfluidic setups that are used for vascular cell interaction studies. For example, Song et al. [38] studied the interaction between endothelial cells and circulating tumor cells, a process that is important for cancer metastasis. They developed a device in which a layer of endothelial cells can be stimulated with chemokines from the bottom, while being treated simultaneously with a suspension of breast cancer cells from the top. When the endothelium was stimulated with CXCL12, a chemokine implicated in metastasis, they found that more cancer cells adhered to the layer of endothelial cells than under basal conditions. Another study on metastasis used a microfluidic chip with small, gel-coated gaps, overlaid with a monolayer of endothelial cells to mimic the basement membrane and the endothelium, respectively [39]. Using this microfluidic in vitro model of a blood vessel, tumor cell migration could be quantified and studied in great detail with time-lapse microscopy. It is not just interactions between tumor cells and endothelium that are an interesting object of research in vascular science. Studying the interactions between other circulating cells and endothelial cells is also important. For example, the binding of leukocytes to endothelial cells is an essential step in inflammation [40], while the endothelium-mediated activation of blood platelets is important in clotting and thrombosis [41]. Multiple reports have been published by groups that studied the adhesion of leukocytes [42] or platelets [43-45] to endothelial cells or endothelial cellderived adhesion factors in microfluidic devices. These reports show that microfluidic cell interaction studies require less sample and reagents than similar, conventional studies. Moreover, a number of these studies already show increased throughput by using parallel channels in one device $[42,44$, 45].

3.4. Three-Dimensional Culturing. An important factor in vascular cell physiology is its three-dimensional microenvironment. Cells are embedded in an environment that comprises other cells, extracellular matrix proteins, bodily fluids, and blood. Three-dimensional cell culturing in the laboratory can be performed by incorporating cells in a hydrogel matrix (e.g., the commercially available, collagenbased Matrigel), or by growing cells on top of this matrix, allowing them to migrate into the gel [46]. Still, the complex real-life, three-dimensional microenvironment is usually reduced to a two-dimensional system when experiments are carried out on cells in vitro. This is more convenient, because with such a system cells can easily be supplied with fresh growth medium, growth factors, and 


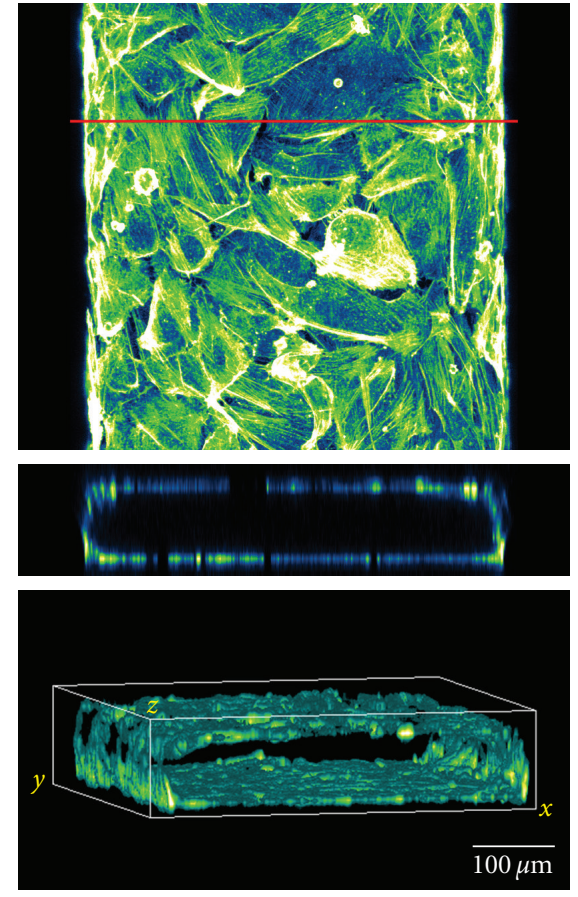

Figure 6: Covering all surfaces of a microfluidic channel yields "artificial capillaries." Human endothelial cells were cultured in a PDMS microfluidic channel and allowed to cover all surfaces. After overnight culturing, cells were fixated and actin filaments were imaged with confocal laser scanning microscopy. Top image is a pseudocolored top view of the microfluidic channel. The red line marks the section that was used to construct a front view of the channel (middle image). The bottom image is an isometric volume view of the same channel.

other soluble compounds. Moreover, a two-dimensional setup is more compatible with microscopy and imaging. However, when using microfluidic devices, replenishment of medium, generation of gradients, and microscopic imaging is relatively easy to realize in a three-dimensional culturing environment. A good example is the recent publication by Vickerman et al. [47] They describe a microfluidic device with two parallel channels, connected by a gel chamber. The gel chamber is filled with a collagen-based hydrogel and endothelial cells are grown in one of the channels. By generating a gradient of soluble growth factors, the endothelial cells grow into the gel and even form open capillaries that span the entire gel chamber from channel to channel. In this particular article, the gel is pipetted into the gel chamber by microinjection before assembling the device. However, using the laminar flow properties discussed earlier in this review, hydrogels can also be formed in situ and even be patterned and confined to certain regions of the microfluidic device [48]. The great potential of these three-dimensional culturing techniques was recently underlined by Barkefors et al. [49], who cultured ex vivo kidney tissue and followed the formation of blood vessels in response to a VEGF gradient. Because of the small scale of microfluidic devices, it is possible to advance this proofof-concept study towards high-throughput assays in order to screen for compounds that affect blood vessel formation in such realistic models. A good example of this highthroughput trend is the recent study by Hsiao et al. [50], who studied three-dimensional, spheroid cocultures of prostate cancer cells and endothelial cells in a microfluidic device with 28 side chambers that could all harbor a tumorous spheroid.

3.5. Compound Screening Assays. In biomedical engineering, a lot of research is dedicated to developing particle systems that carry drugs, proteins, DNA for gene therapy or siRNA for gene silencing to their proper site of action. In this field of research, it is important to have a way to quickly screen for adhesion to endothelial cells - the first barrier that particles encounter when injected intravenously. Screening under static conditions in well plates ignores the mechanical forces caused by the flowing blood, which counter particle adhesion. A recent study by our group, using fluorescent siRNA-containing polymer particles, shows that microfluidic technology allows for quick screening of particle adhesion to endothelial cells under dynamic conditions [51]. More realistic microfluidic models of microvasculature have been developed by Prabhakarpandian et al. [52] for the same purposes. These devices contain channels that are designed after real capillary networks. They show that capillary geometry has a strong influence on local mechanical conditions and particle adhesion. The fabrication of a more complex microfluidic device that tries to mimic the tight blood-brain barrier, while still being easy-to-use in high-throughput assays, was recently reported by Genes et al. [53]. It is to be expected that high-throughput screening in these more realistic vascular models of the in vivo situation will become the norm in drug development and material science in the future.

3.6. Stem Cells and Tissue Engineering. Regenerative medicine is the field in which researchers try to engineer tissues in the laboratory to replace damaged or missing tissue in the human body. It is a multi disciplinary field, which combines materials science with cell biology and biomedicine. A major challenge in this field is the production of vascularized tissue for implantation. In order to achieve this, stem cells must be stimulated to differentiate into vascular cells, and these vascular cells need to arrange themselves into a vascular network. Microfluidic technology can be of use in both processes. For differentiation of human stem cells to vascular tissue, many factors can be of influence. Because human stem cells and the inducing factors are relatively difficult to obtain, it is advantageous to perform tests in a microfluidic setting instead of in a macroscopic assay. Figallo et al. [54] developed a 12-well micro-bioreactor in which human embryonic stem cells were directed towards a vascular phenotype by varying growth factors, perfusion, and cell seeding density. Using such microfluidic devices instead of conventional techniques saves reagents and allows for more flexibility in terms of culture parameters. 
The other aspect of vascular tissue engineering in which microfluidic technology can be of help is in preparing vascular networks that can be incorporated into tissue constructs. This can be accomplished by two approaches. First of all, a "synthetic capillary" can be engineered by using microfluidic technology. This works by designing a microfluidic channel of which the walls contain tiny gaps of only a few micrometer in diameter and tens of micrometers in length. Behind these gaps, compartments are located in which tissue can be grown. When medium is pumped through the channel, the gaps act as a simple endothelium-like barrier, limiting mass transport to the tissue compartments. An example of such a microfluidic design was reported by Lee et al. [55], who used this principle to design synthetic analogs of liver sinusoids. Using this approach, mass transport over the endothelium-like barrier can be tweaked to mimic the values found in human vessels [56, 57]. A second approach to use microfluidic technology for generation of vascular networks is based on the notion that the microfluidic device itself can be considered as a three-dimensional "scaffold" in which cells can be grown. When all sides of a channel are completely covered with endothelial cells, microvascular networks are generated that mimic in vivo networks (Figure 6). It has been shown that this approach will in principle work: PDMS devices with microvascular network morphologies can be completely covered with endothelial cells to generate capillary-like structures [58]. However, PDMS is a nonbiodegradable polymer. Biodegradability is paramount if eventually the material is to be replaced with functional tissue. Another study has shown that the same type of system can also be built with the biodegradable polymer poly (glycerol sebacate) [59]. Still, these microfluidic devices consist of only one flat layer of vascular structures. A major challenge will be to build biodegradable, truly three-dimensional microvascular networks that can be combined with other materials and cells in regenerative medicine. Novel rapid techniques for three-dimensional device fabrication, such as stereolithography with biodegradable polymers [60], hold great promise to overcome this challenge.

\section{Conclusion}

The examples given in this review clearly illustrate the fact that the use of microfluidic technology facilitates current vascular research and, more importantly, opens up novel areas of research that are not possible with more conventional setups and techniques. It is important to realize not only that microfluidic technology paves the way for more realistic in vitro models in vascular cell biology but also that the technology is still in its infancy in terms of throughput. Almost all studies described in this review are proof-ofprinciple experiments that require a lot of personal effort and intervention by the researcher. However, automation, standardization, and increasing scale will all be natural stages in the maturation of microfluidic technology. These improvements will boost the systematic nature of vascular cell biological research in the future.

\section{Acknowledgment}

The authors would like to thank the two anonymous reviewers for their useful suggestions and comments on the original manuscript.

\section{References}

[1] M. Félétou and P. M. Vanhoutte, "Endothelium-derived hyperpolarizing factor: where are we now?" Arteriosclerosis, Thrombosis, \& Vascular Biology, vol. 26, no. 6, pp. 1215-1225, 2006.

[2] K. Ley, C. Laudanna, M. I. Cybulsky, and S. Nourshargh, "Getting to the site of inflammation: the leukocyte adhesion cascade updated," Nature Reviews Immunology, vol. 7, no. 9, pp. 678-689, 2007.

[3] J. Folkman, "Angiogenesis in cancer, vascular, rheumatoid and other disease," Nature Medicine, vol. 1, no. 1, pp. 27-31, 1995.

[4] E. Durand, A. Scoazec, A. Lafont, et al., "In vivo induction of endothelial apoptosis leads to vessel thrombosis and endothelial denudation: a clue to the understanding of the mechanisms of thrombotic plaque erosion," Circulation, vol. 109, no. 21, pp. 2503-2506, 2004.

[5] S. Kim and H. von Recum, "Endothelial stem cells and precursors for tissue engineering: cell source, differentiation, selection, and application," Tissue Engineering. Part B, vol. 14, no. 1, pp. 133-147, 2008.

[6] J. Davignon and P. Ganz, "Role of endothelial dysfunction in atherosclerosis," Circulation, vol. 109, no. 23, supplement 1, pp. 27-32, 2004.

[7] D. J. Hicklin and L. M. Ellis, "Role of the vascular endothelial growth factor pathway in tumor growth and angiogenesis," Journal of Clinical Oncology, vol. 23, no. 5, pp. 1011-1027, 2005.

[8] F. Cosentino and T. F. Luscher, "Endothelial dysfunction in diabetes mellitus," Journal of Cardiovascular Pharmacology, vol. 32, supplement 3, pp. S54-S61, 1998.

[9] Y.-S. Li, J. H. Haga, and S. Chien, "Molecular basis of the effects of shear stress on vascular endothelial cells," Journal of Biomechanics, vol. 38, no. 10, pp. 1949-1971, 2005.

[10] J. H. Haga, Y.-S. Li, and S. Chien, "Molecular basis of the effects of mechanical stretch on vascular smooth muscle cells," Journal of Biomechanics, vol. 40, no. 5, pp. 947-960, 2007.

[11] G. E. Davis and D. R. Senger, "Endothelial extracellular matrix: biosynthesis, remodeling, and functions during vascular morphogenesis and neovessel stabilization," Circulation Research, vol. 97, no. 11, pp. 1093-1107, 2005.

[12] E. Dejana, "Endothelial cell-cell junctions: happy together," Nature Reviews Molecular Cell Biology, vol. 5, no. 4, pp. 261270, 2004.

[13] Z. M. Ruggeri, "Von Willebrand factor, platelets and endothelial cell interactions," Journal of Thrombosis and Haemostasis, vol. 1, no. 7, pp. 1335-1342, 2003.

[14] E. Paternotte, C. Gaucher, P. Labrude, J.-F. Stoltz, and P. Menu, "Review: behaviour of endothelial cells faced with hypoxia," Bio-Medical Materials and Engineering, vol. 18, no. 4-5, pp. 295-299, 2008.

[15] R. M. Rao, L. Yang, G. Garcia-Cardena, and F. W. Luscinskas, "Endothelial-dependent mechanisms of leukocyte recruitment to the vascular wall," Circulation Research, vol. 101, no. 3, pp. 234-247, 2007.

[16] J. El-Ali, P. K. Sorger, and K. F. Jensen, "Cells on chips," Nature, vol. 442, no. 7101, pp. 403-411, 2006. 
[17] I. Meyvantsson and D. Beebe, "Cell culture models in microfluidic systems," Annual Review of Analytical Chemistry, vol. 1, no. 1, pp. 423-449, 2008.

[18] D. C. Duffy, J. C. McDonald, O. J. Schueller, and G. M. Whitesides, "Rapid prototyping of microfluidic systems in poly(dimethylsiloxane)," Analytical Chemistry, vol. 70, no. 23, pp. 4974-4984, 1998.

[19] L. Kim, Y.-C. Toh, J. Voldman, and H. Yu, "A practical guide to microfluidic perfusion culture of adherent mammalian cells," Lab on a Chip, vol. 7, no. 6, pp. 681-694, 2007.

[20] S. Takayama, E. Ostuni, P. LeDuc, K. Naruse, D. E. Ingber, and G. M. Whitesides, "Subcellular positioning of small molecules," Nature, vol. 411, no. 6841, p. 1016, 2001.

[21] D. M. Thompson, K. R. King, K. J. Wieder, M. Toner, M. L. Yarmush, and A. Jayaraman, "Dynamic gene expression profiling using a microfabricated living cell array," Analytical Chemistry, vol. 76, no. 14, pp. 4098-4103, 2004.

[22] N. V. Tolan, L. I. Genes, W. Subasinghe, M. Raththagala, and D. M. Spence, "Personalized metabolic assessment of erythrocytes using microfluidic delivery to an array of luminescent wells," Analytical Chemistry, vol. 81, no. 8, pp. 3102-3108, 2009.

[23] C. Situma, M. Hashimoto, and S. A. Soper, "Merging microfluidics with microarray-based bioassays," Biomolecular Engineering, vol. 23, no. 5, pp. 213-231, 2006.

[24] K. S. Cunningham and A. I. Gotlieb, "The role of shear stress in the pathogenesis of atherosclerosis," Laboratory Investigation, vol. 85, no. 1, pp. 9-23, 2005.

[25] J. W. Song, W. Gu, N. Futai, K. A. Warner, J. E. Nor, and S. Takayama, "Computer-controlled microcirculatory support system for endothelial cell culture and shearing," Analytical Chemistry, vol. 77, no. 13, pp. 3993-3999, 2005.

[26] E. Tkachenko, E. Gutierrez, M. H. Ginsberg, and A. Groisman, "An easy to assemble microfluidic perfusion device with a magnetic clamp," Lab on a Chip, vol. 9, no. 8, pp. 1085-1095, 2009.

[27] M. J. Kuchan and J. A. Frangos, "Role of calcium and calmodulin in flow-induced nitric oxide production in endothelial cells," American Journal of Physiology, vol. 266, no. 3, pp. C628-C636, 1994.

[28] D. M. Spence, N. J. Torrence, M. L. Kovarik, and R. S. Martin, "Amperometric determination of nitric oxide derived from pulmonary artery endothelial cells immobilized in a microchip channel," The Analyst, vol. 129, no. 11, pp. 9951000, 2004.

[29] T. D. Oblak, P. Root, and D. M. Spence, "Fluorescence monitoring of ATP-stimulated, endothelium-derived nitric oxide production in channels of a poly(dimethylsiloxane)based microfluidic device," Analytical Chemistry, vol. 78, no. 9, pp. 3193-3197, 2006.

[30] E. W. Young, A. R. Wheeler, and C. A. Simmons, "Matrixdependent adhesion of vascular and valvular endothelial cells in microfluidic channels," Lab on a Chip, vol. 7, no. 12, pp. 1759-1766, 2007.

[31] S. Usami, H.-H. Chen, Y. Zhao, S. Chien, and R. Skalak, "Design and construction of a linear shear stress flow chamber," Annals of Biomedical Engineering, vol. 21, no. 1, pp. 7783, 1993.

[32] I. Barkefors, S. Le Jan, L. Jakobsson, et al., "Endothelial cell migration in stable gradients of vascular endothelial growth factor A and fibroblast growth factor 2: effects on chemotaxis and chemokinesis," Journal of Biological Chemistry, vol. 283, no. 20, pp. 13905-13912, 2008.
[33] N. Zaari, P. Rajagopalan, S. K. Kim, A. J. Engler, and J. Y. Wong, "Photopolymerization in microfluidic gradient generators: microscale control of substrate compliance to manipulate cell response," Advanced Materials, vol. 16, no. 23-24, pp. 2133 2137, 2004.

[34] F.-Q. Nie, M. Yamada, J. Kobayashi, M. Yamato, A. Kikuchi, and T. Okano, "On-chip cell migration assay using microfluidic channels," Biomaterials, vol. 28, no. 27, pp. 4017-4022, 2007.

[35] C.-C. Wu, Y.-S. Li, J. H. Haga, et al., "Directional shear flow and Rho activation prevent the endothelial cell apoptosis induced by micropatterned anisotropic geometry," Proceedings of the National Academy of Sciences of the United States of America, vol. 104, no. 4, pp. 1254-1259, 2007.

[36] D. T. Chiu, N. L. Jeon, S. Huang, et al., "Patterned deposition of cells and proteins onto surfaces by using three-dimensional microfluidic systems," Proceedings of the National Academy of Sciences of the United States of America, vol. 97, no. 6, pp. 24082413, 2000.

[37] S. Takayama, J. C. Mcdonald, E. Ostuni, et al., "Patterning cells and their environments using multiple laminar fluid flows in capillary networks," Proceedings of the National Academy of Sciences of the United States of America, vol. 96, no. 10, pp. 5545-5548, 1999.

[38] J. W. Song, S. P. Cavnar, A. C. Walker, et al., "Microfluidic endothelium for studying the intravascular adhesion of metastatic breast cancer cells," PLoS ONE, vol. 4, no. 6, 2009.

[39] K. C. Chaw, M. Manimaran, E. H. Tay, and S. Swaminathan, "Multi-step microfluidic device for studying cancer metastasis," Lab on a Chip, vol. 7, no. 8, pp. 1041-1047, 2007.

[40] R. Alon and S. Feigelson, "From rolling to arrest on blood vessels: leukocyte tap dancing on endothelial integrin ligands and chemokines at sub-second contacts," Seminars in Immunology, vol. 14, no. 2, pp. 93-104, 2002.

[41] P. Andre, C. V. Denis, J. Ware, et al., "Platelets adhere to and translocate on von Willebrand factor presented by endothelium in stimulated veins," Blood, vol. 96, no. 10, pp. 3322-3328, 2000.

[42] U. Y. Schaff, M. M. Q. Xing, K. K. Lin, N. Pan, N. L. Jeon, and S. I. Simon, "Vascular mimetics based on microfluidics for imaging the leukocyte-endothelial inflammatory response," Lab on a Chip, vol. 7, no. 4, pp. 448-456, 2007.

[43] K. B. Neeves and S. L. Diamond, "A membrane-based microfluidic device for controlling the flux of platelet agonists into flowing blood," Lab on a Chip, vol. 8, no. 5, pp. 701-709, 2008.

[44] C. J. Ku, T. D. Oblak, and D. M. Spence, "Interactions between multiple cell types in parallel microfluidic channels: monitoring platelet adhesion to an endothelium in the presence of an anti-adhesion drug," Analytical Chemistry, vol. 80, no. 19, pp. 7543-7548, 2008.

[45] E. Gutierrez, B. G. Petrich, S. J. Shattil, M. H. Ginsberg, A. Groisman, and A. Kasirer-Friede, "Microfluidic devices for studies of shear-dependent platelet adhesion," Lab on a Chip, vol. 8, no. 9, pp. 1486-1495, 2008.

[46] B. Vailhé, D. Vittet, and J.-J. Feige, "In vitro models of vasculogenesis and angiogenesis," Laboratory Investigation, vol. 81, no. 4, pp. 439-452, 2001.

[47] V. Vickerman, J. Blundo, S. Chung, and R. D. Kamm, "Design, fabrication and implementation of a novel multi-parameter control microfluidic platform for three-dimensional cell culture and real-time imaging," Lab on a Chip, vol. 8, no. 9, pp. 1468-1477, 2008. 
[48] M. S. Kim, J. H. Yeon, and J.-K. Park, "A microfluidic platform for 3-dimensional cell culture and cell-based assays," Biomedical Microdevices, vol. 9, no. 1, pp. 25-34, 2007.

[49] I. Barkefors, S. Thorslund, F. Nikolajeff, and J. Kreuger, "A fluidic device to study directional angiogenesis in complex tissue and organ culture models," Lab on a Chip, vol. 9, no. 4, pp. 529-535, 2009.

[50] A. Y. Hsiao, Y.-S. Torisawa, Y.-C. Tung, et al., "Microfluidic system for formation of PC-3 prostate cancer co-culture spheroids," Biomaterials, vol. 30, no. 16, pp. 3020-3027, 2009.

[51] A. D. van der Meer, M. M. J. Kamphuis, A. A. Poot, J. J. Feijen, and I. Vermes, "A microfluidic device for monitoring siRNA delivery under fluid flow," Journal of Controlled Release, vol. 132, no. 3, pp. 42-44, 2008.

[52] B. Prabhakarpandian, K. Pant, R. C. Scott, et al., "Synthetic microvascular networks for quantitative analysis of particle adhesion," Biomedical Microdevices, vol. 10, no. 4, pp. 585595, 2008.

[53] L. I. Genes, N. V. Tolan, M. K. Hulvey, R. S. Martin, and D. M. Spence, "Addressing a vascular endothelium array with blood components using underlying microfluidic channels," Lab on a Chip, vol. 7, no. 10, pp. 1256-1259, 2007.

[54] E. Figallo, C. Cannizzaro, S. Gerecht, et al., "Micro-bioreactor array for controlling cellular microenvironments," Lab on a Chip, vol. 7, no. 6, pp. 710-719, 2007.

[55] P. J. Lee, P. J. Hung, and L. P. Lee, "An artificial liver sinusoid with a microfluidic endothelial-like barrier for primary hepatocyte culture," Biotechnology \& Bioengineering, vol. 97, no. 5, pp. 1340-1346, 2007.

[56] M. Khakpour and K. Vafai, "A comprehensive analytical solution of macromolecular transport within an artery," International Journal of Heat and Mass Transfer, vol. 51, no. 11-12, pp. 2905-2913, 2008.

[57] M. Khakpour and K. Vafai, "Critical assessment of arterial transport models," International Journal of Heat and Mass Transfer, vol. 51, no. 3-4, pp. 807-822, 2008.

[58] M. Shin, K. Matsuda, O. Ishii, et al., "Endothelialized networks with a vascular geometry in microfabricated poly(dimethyl siloxane)," Biomedical Microdevices, vol. 6, no. 4, pp. 269-278, 2004.

[59] C. Fidkowski, M. R. Kaazempur-Mofrad, J. Borenstein, J. P. Vacanti, R. Langer, and Y. Wang, "Endothelialized microvasculature based on a biodegradable elastomer," Tissue Engineering, vol. 11, no. 1-2, pp. 302-309, 2005.

[60] S. M. Peltola, F. P. W. Melchels, D. W. Grijpma, and M. Kellomäki, "A review of rapid prototyping techniques for tissue engineering purposes," Annals of Medicine, vol. 40, no. 4, pp. 268-280, 2008. 

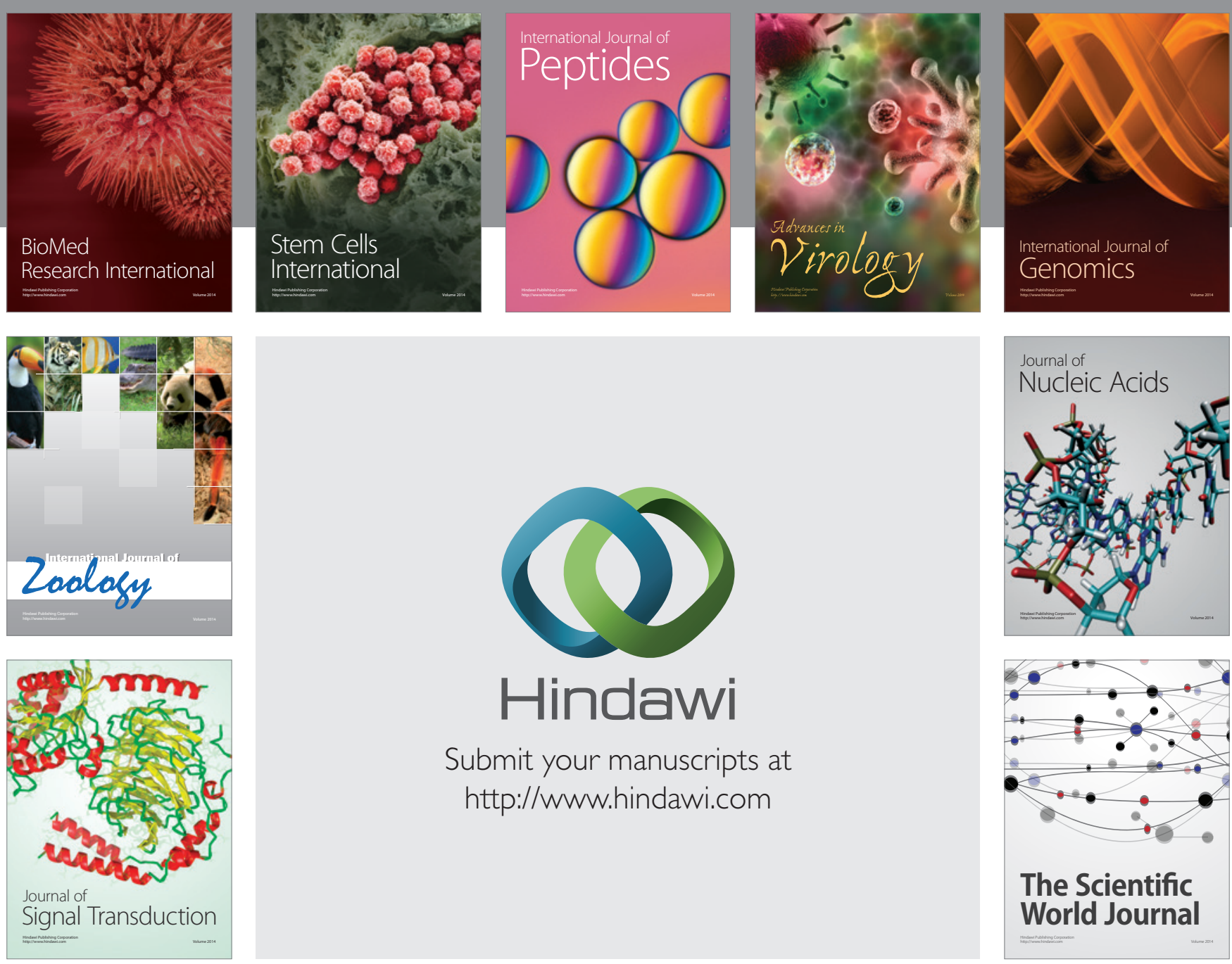

Submit your manuscripts at

http://www.hindawi.com
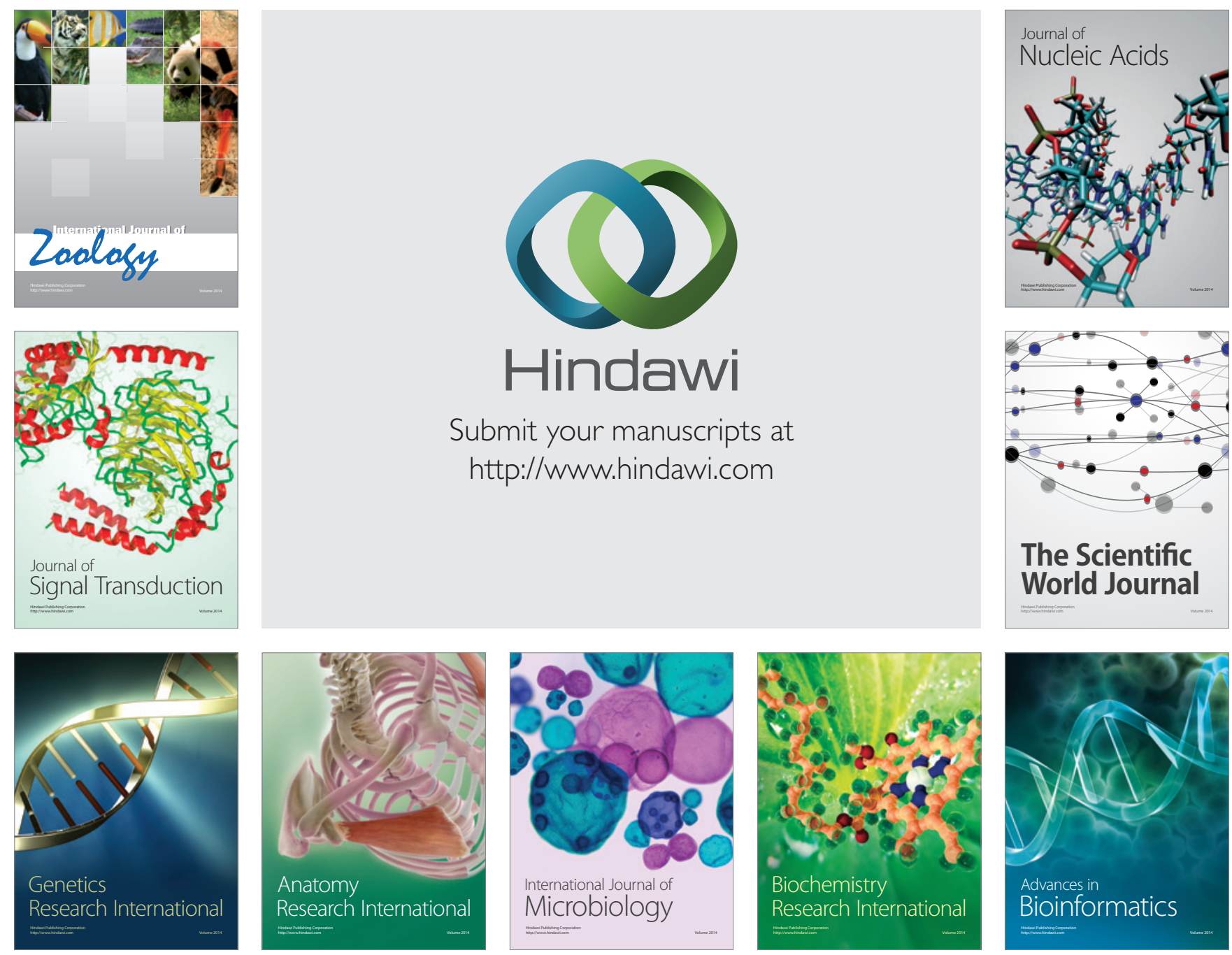

The Scientific World Journal
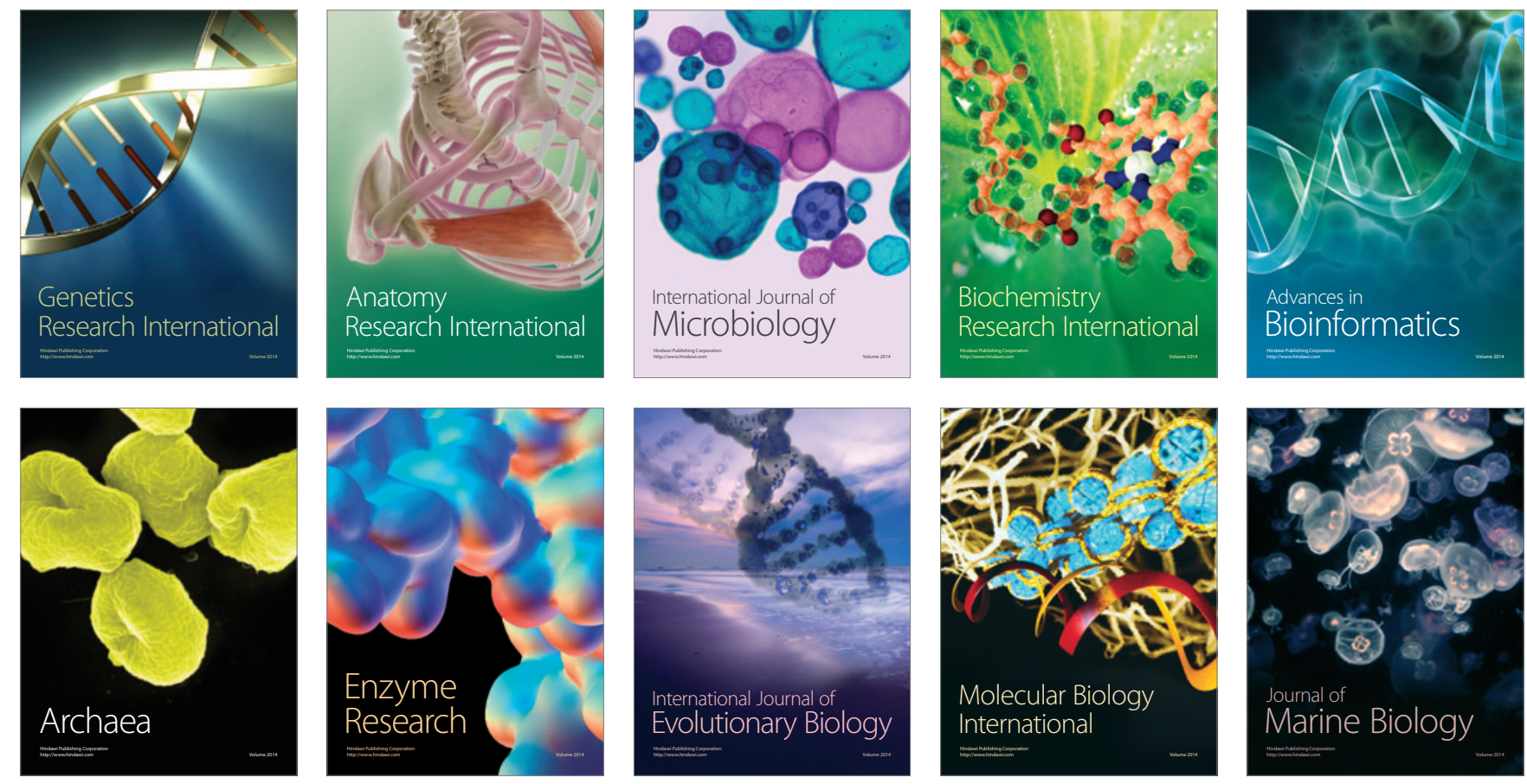